\title{
Analysis of IGP Routing Protocols for Real Time Applications: A Comparative Study
}

\author{
Mohamad A. Yehia \\ British University in Egypt \\ Suez Desert Road \\ El Sherouk City-Cairo
}

\author{
Mohammed S. Aziz \\ British University in Egypt Suez \\ Desert Road \\ El Sherouk City-Cairo
}

\author{
Hussein A. Elsayed \\ British University in Egypt Suez \\ Desert Road \\ El Sherouk City-Cairo
}

\begin{abstract}
This paper describes the subject of dynamic routing protocols performance of EIGRP, OSPF and RIP in real time applications. Furthermore the effect of dividing OSPF networks into several areas in real time applications will be discussed.

Problems facing large networks are the increased routed traffic and the reduction in stability of the network, moreover the security of the network that can be easily breached the size increase.

In this paper the performance parameters that determine the performance of RIP, OSPF, and EIGRP was evaluated along with studying the effect of using areas in OSPF as a routing protocol. These parameters are: convergence duration, traffic sent, End to End delay and delay variation, utilization, and packet loss.

As a specific interest, we are going to study the effect of OSPF areas on the quality of service of voice over IP as an application layer level. [1].

OPNET simulation tool is used to compare different routing protocols besides comparing different area division topologies and measuring different performance parameters.

this paper consist of two parts, the first analyze the performance of EIGRP,RIP,OSPF and the second part focus on OSPF areas since OSPF is the most common routing protocol.
\end{abstract}

\section{Keywords}

RIP, EIGRP, OSPF, OSPF areas, OPNET, Delay variation, End to End delay

\section{INTRODUCTION}

Nowadays routing protocols became a vital role in modern network communications as they are the means of information propagation between routers in the network. They also determine the route for communication with each other. Then the packets are forwarded through the optimum path according to the algorithm used for each one. [2].

Simply by definition a routing protocol is a set of rules that communication networks follows when computers try to communicate with each other across networks. Routed protocols are the protocol that is responsible for the transportation of data of applications across a network such as IP. Routing protocols are the software that allow routers to dynamically advertise and learn routes, determine which routes are available and which are the most efficient routes to a destination in other words with the best metrics value [3]. Routing protocols are either interior or exterior gateway protocols (IGP) and (EGP) respectively. RIP, OSPF, EIGRP are examples of IGP while BGP and BGP4 are examples of BGP. [4]

Routing protocols are based on various algorithms which in turn depend on metrics to find the best path for sending data. Examples of metrics cost and bandwidth etc. These metrics are stored in routing tables which are continuously updated by the dynamic routing protocols [5].

Currently Open Shortest Path First (OSPF) is the most commonly used and promising intra-domain internet routing protocol thus OSPF areas will be discussed in details. In OSPF, packets are routed along shortest paths to the destination. OSPF is a link-state routing protocol. It is designed to run in a single Autonomous System (AS). Each OSPF router keeps the same database describing the Autonomous System's topology. From this database, a routing table is calculated by constructing a shortest path tree. When the topology changes, OSPF will recalculate routes quickly to utilize the minimum routing protocol traffic. An area routing capability is provided, enabling an additional level of routing protection and a reduction in routing protocol traffic [6].

\section{PROTOCOL OVERVIEW}

RIP protocol uses distance-vector algorithm where it finds the best path to a remote network by judging distance. The route having the least number of hops to the destination is determined to be the best route. It sends the entire routing table to directly connected neighbors. The only routing metric RIP uses is hop count; therefore, it does not necessarily select the fastest path to a destination [7].

EIGRP which is the Enhanced Interior Gateway Routing Protocol is a kind of Hybrid protocols where it uses aspects of both distance vector and link state. It is one of the protocols that use the hybrid algorithm and was developed to address the problems associated with routing in large networks that are beyond the RIP scope. Moreover, it selects the fastest path based on the bandwidth, delay, reliability and load. By default, it uses only bandwidth and delay metrics [8].

OSPF, open shortest-path-first protocol is a link state routing protocol. The link state protocol sends updates containing the state of their own links to all other routers on the network. Each router creates three separate tables. One of these tables keeps track of directly attached neighbors, one determines the topology of the entire internetwork, and one is used as the routing table. Link state routers know more about the internetwork than any distancevector routing protocol [9].

OSPF can be configured to different areas. Areas can be connected to the back bone in two ways, either by direct physical link or by virtual link that goes through other intermediate areas called, "Transient area".

In a very large AS with a large number of networks, each OSPF router must keep the LSA (link state advertisement) of every other router in its LSDB (Link state data base). The Shortest path first calculations of a large LSDB require an extensive amount of 
processing. Also, the resulting routing table is very large, containing a route to each network in the AS. [10]

In an attempt to shrink the size of the LSDB and the processing overhead for the SPF tree and routing table calculation, OSPF allows the AS to be divided up into adjacent groups of networks called areas. Areas are identified through a 32-bit Area ID expressed in dotted decimal notation [11].

This availability of this type of configuration offers a lot of security. Moreover it reduces the amount of routed traffic through the network especially at bottle necks.

To keep the size of the LSDB for each router to a minimum, LSAs for an area's networks and routers are flooded within the area but not to routers outside the area. Thus each area becomes its own link state domain with its own LSDB [11].

If a router is connected to multiple areas, it has multiple LSDBs and SPF Trees. The routing table is a combination of the routing table entries of all the SPF Trees for the router as well as static routes, and routes learned from other routing protocols [2].

To minimize the number of entries in the routing tables of OSPF routers, the networks inside an area are advertised outside the area using summary route advertisements through ABR (Area border router). Using route summarization, the topology (the networks and their path costs) of an area is unseen from the rest of the AS [4] [12].

When the topology of an area is hidden, the rest of the AS is protected from route flapping and events that cause networks to unstablize. If a network comes up, the event is propagated as a Link State Update and flooded within the area through adjacencies to routers. However, because all the networks inside the area are advertised outside the area using summary routes, the Link State Update is not flooded outside the area [4]

\section{PARAMETERS DEFINITIONS}

\section{Convergence duration}

It Records the time it takes the Protocol routing table on this router to convergence. The convergence time is the time elapsed since a first sign of convergence activity occurs until a sign of convergence activity occurs that is followed by a certain interval of no convergence activity.

\section{End to end delay}

It is the Variance among end to end delays for voice packets. End to end delay for a voice packet is measured from the time it is created to the time it is received [4].

\section{Delay variation}

The total voice packet delay, called "analog-to-analog" or "mouthto-ear" delay is $=($ network delay $)+($ encoding delay $)+($ decoding delay $)+($ compression delay $)+($ decompression delay $)$. [13]

Network delay is the time at which the sender node gave the packet to RTP to the time the receiver got it from RTP. Encoding delay (on the sender node) is computed from the encoder scheme. Decoding delay (on the receiver node) is assumed to be equal to the encoding delay. Compression and Decompression delays come from the corresponding attributes in the Voice application configuration.

\section{PART 1: RIP, EIGRP, AND OSPF PERFORMANCE}

\subsection{Network Model}

The network model shown in figure 1 shows the network which coveres the Europian continent where in each capital of the 16 chosen countries the node model consists of a router and a LAN network of 20 working stations. The router and the LAN were connected through an Ethernet interface (100Mbps) as in figure 2, while the routers were connected to each other with OC3 fiber cable $(\approx 148$ Mbps). Each router was connected to three routers thus this network is not fully mesh to allow no direct routing information exchange.

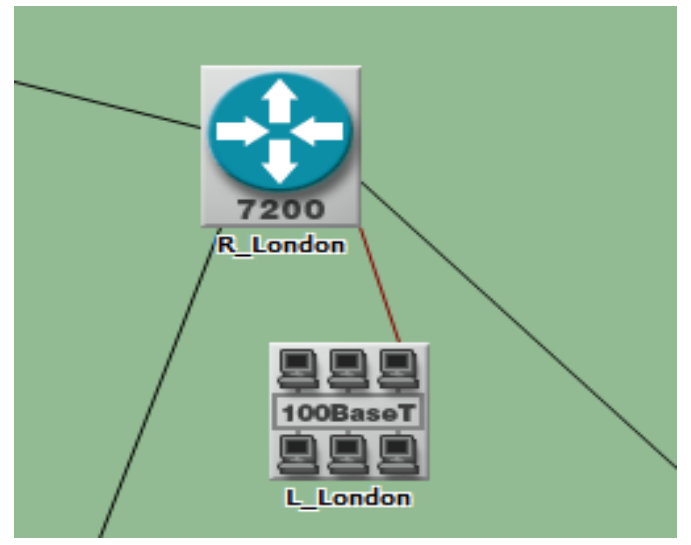

Figure 1: Network Model

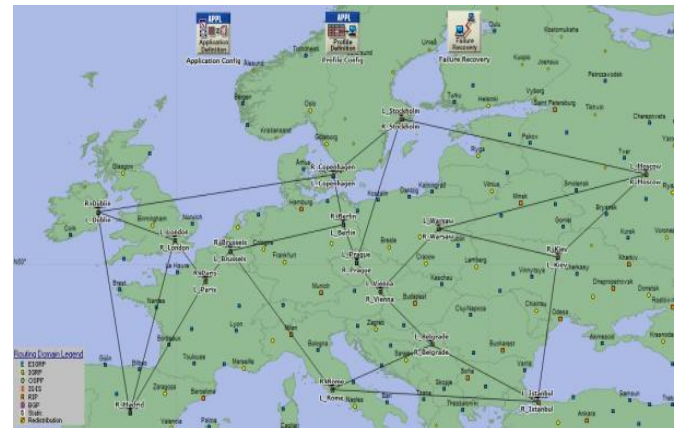

Figure 2 : Node Model

Three applications where used in this network are shown in table 1

Table 1 : Applications Description

\begin{tabular}{|c|c|}
\hline Application & Description \\
\hline Voice & Low Quality Speech \\
\hline HTTP & Light Browsing \\
\hline FTP & Low Load \\
\hline
\end{tabular}

These three applications were added to one profile which was assigned to each working station of the entire network. All three 
applications were simulated to run simultaneously and only one during the simulations time.

\subsection{Simulation results}

In this simulation, the OPNET simulator will be used. OPNET is used to simulate and find network performance in a virtual network that imitates a real network.

The model discussed earlier was simulated for 10 minutes which was a suitable time for all results and graphs obtained. The graphs obtained resulted from two separate simulations the first one was three scenarios of different routing protocol without failure of the Dublin Router while the second simulation contained the same three scenarios of routing protocols but after applying the node failure for Dublin router which was applied after 200 seconds. Each parameter either related to routing protocol or application is clarified by two graphs, one without node failure and the other after applying node failure. Since this paper is discussing the effect on real time applications results were simulated for the voice applications.

Figures 3 shows the conversion time without failure, where it's clear that the EIGRP conversion duration was the least with a value of about 0.02 seconds and the first to converge. Then the RIP protocol was the second one which took about 15 seconds and finally the OSPF which took about 45 seconds.

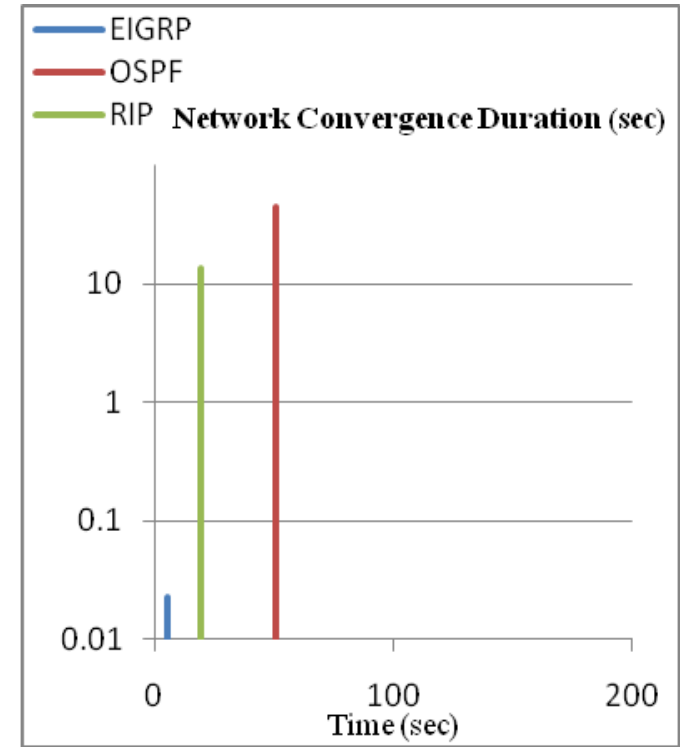

Figure 3 : Netwrok convergence duration

In figure 4 after applying the node failure at 200 seconds the EIGRP was also with the least one in convergence time and the fastest one to respond to the node failure then came the OSPF which took more time and responded lately.

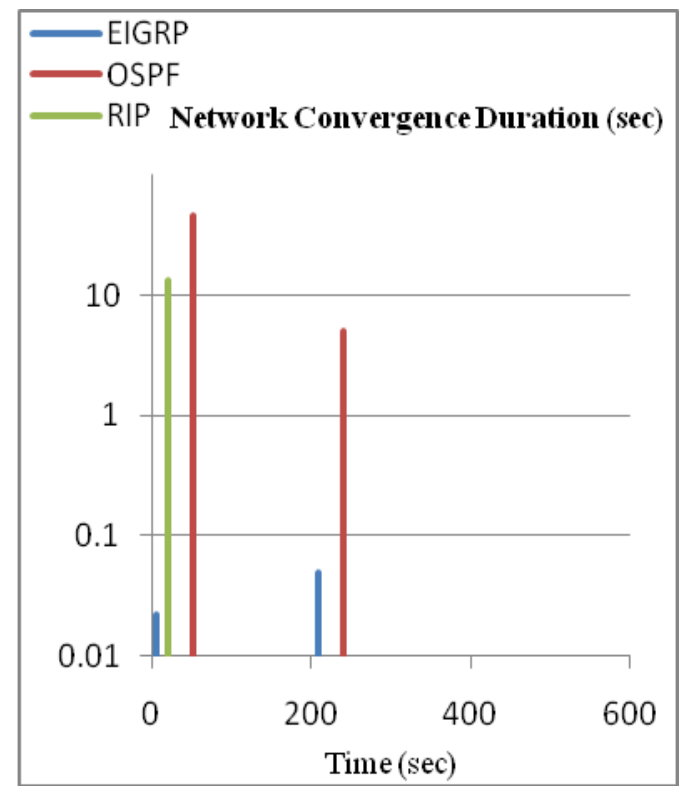

Figure 4 : Network convergence duration after failure

Figure 5 shows than When there was no node failure in the OSPF was the one with the most traffic updates sent due to the packet header size but because of its delay in conversion time it was the last one to send the traffic update. then came the EIGRP which was the first one to send traffic due to the fast convergence time followed by the RIP protocol with the least traffic update sent. In figure 6 after applying the node failure the EIGRP was the fastest to response with traffic sent more than the OSPF which sent traffic after the EIGRP.

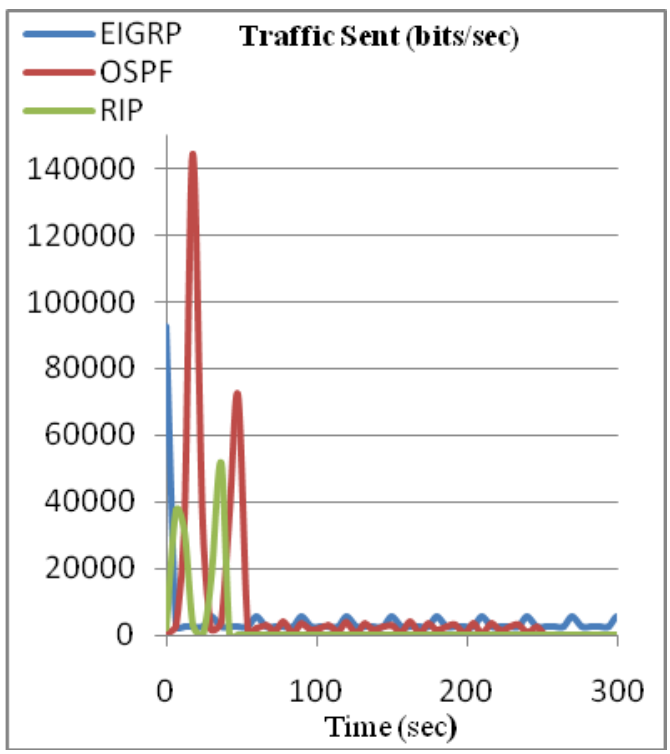

Figure 5 : traffic sent (bits/sec) 


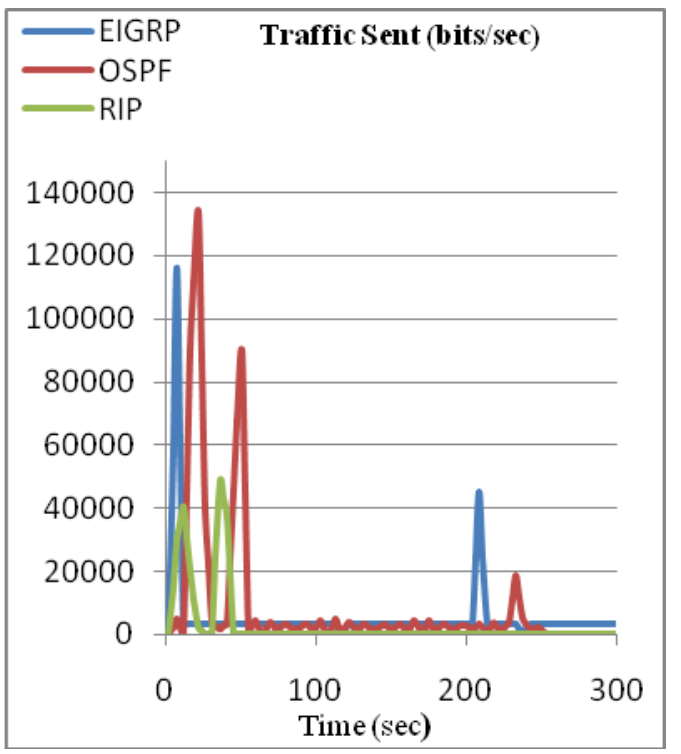

Figure 6 : traffic sent (bits/sec) after failure

In figure 7 the RIP protocol had the biggest delay as it is a simple protocol and is not efficient as the OSPF and EIGRP since it only depends on the hop count metric unlike the others. Then the EIGRP delay was slightly less than the OSPF which both gave better results than the RIP as they both select better route. After applying the node failure in figure 8 the OSPF reaction to the failure was better as the delay became less than the EIGRP but that was after disturbance in the network till it became steady.

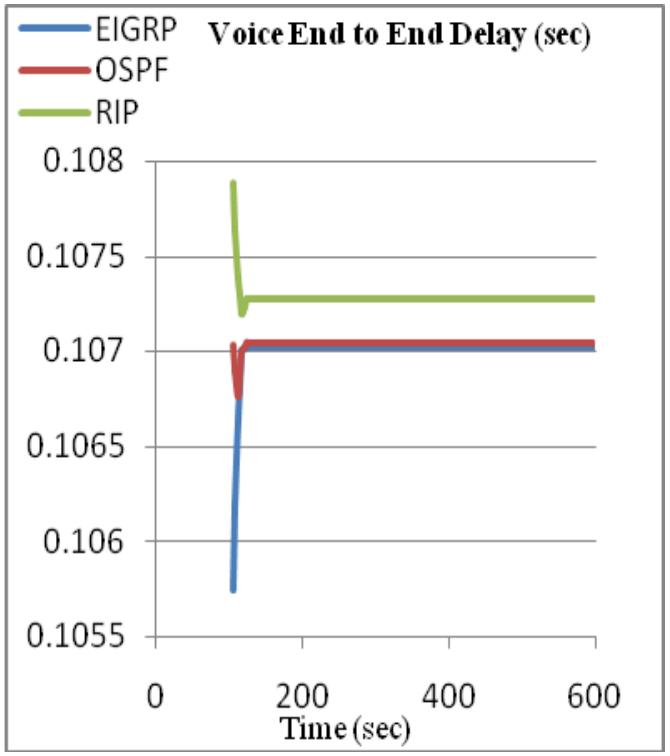

Figure 7 : End to End delay (sec)

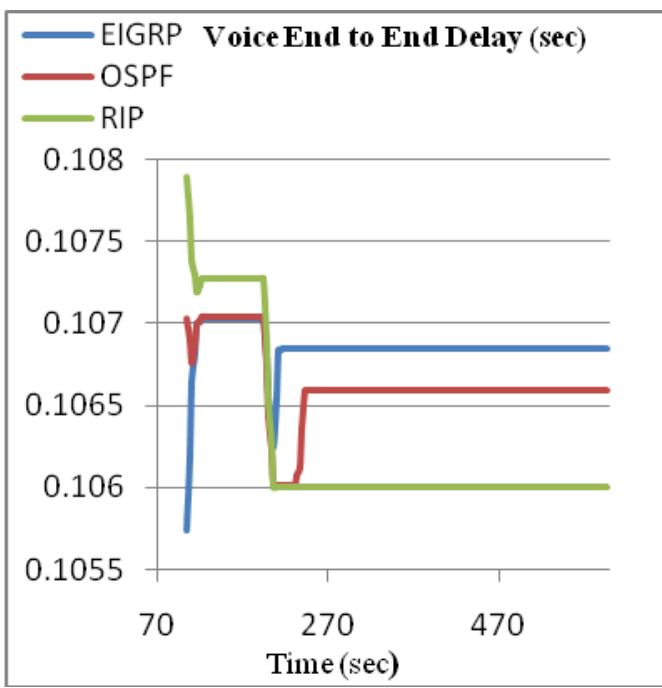

Figure 8 : End to End delay (sec) after failure

In figure 9 the delay variation of OSPF protocol was the highest unlike the EIGRP and RIP which almost had the same delay, but afterwards they all reached a steady state having the same value.

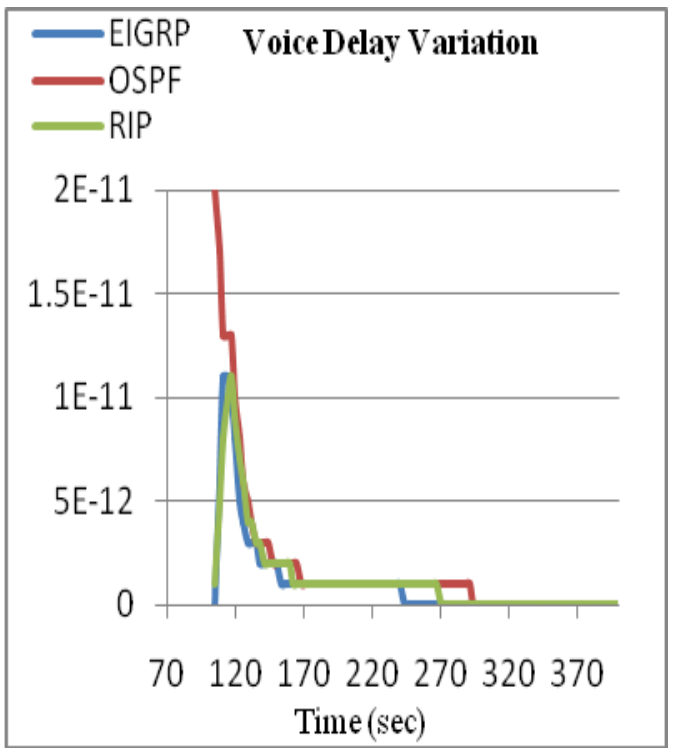

Figure 9 : Voice packet delay variation

In figure 10 the voice traffic received wasn't affected by any of the delay, jitter or delay variation thus all protocols gave the same reading exactly which is about 425,000 bytes/sec. 


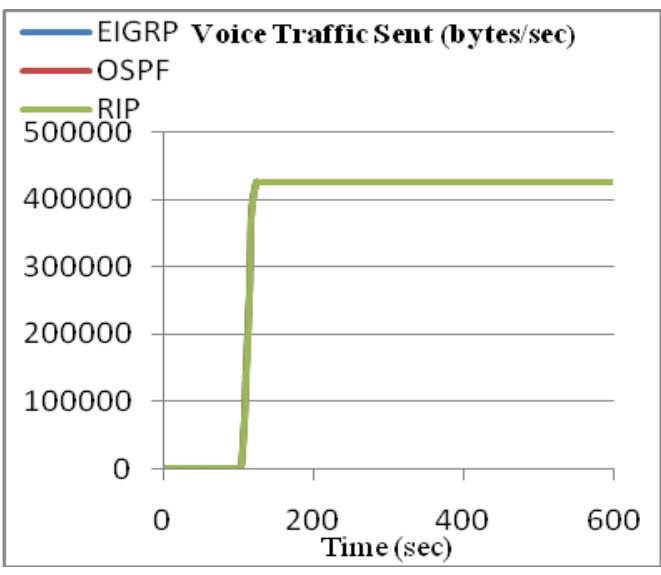

Figure 10 : Voice traffic sent

\section{PART II: OSPF AREA PERFORMANCE}

\subsection{Network model}

The model consists of 16 subnet, each one represent an Egyptian city. Not all Egyptain cities are repsented.

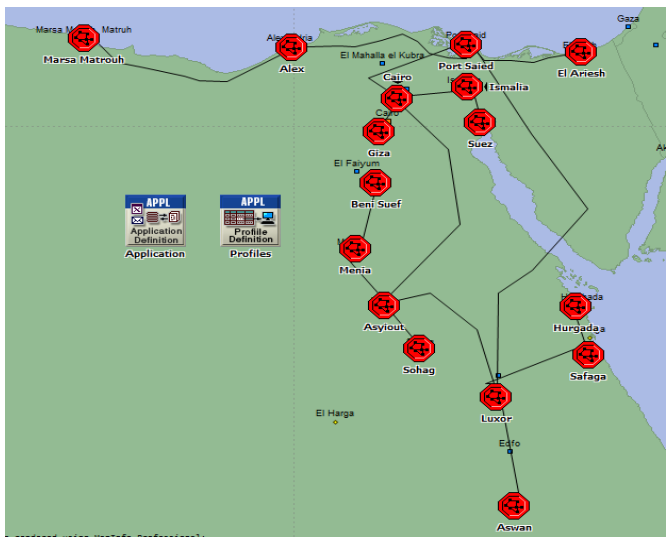

Figure 11 : Model under test

Each subnet consists of five Cisco routers (7206) and 9 Ethernet Workstations (PC).they are connected as follows:

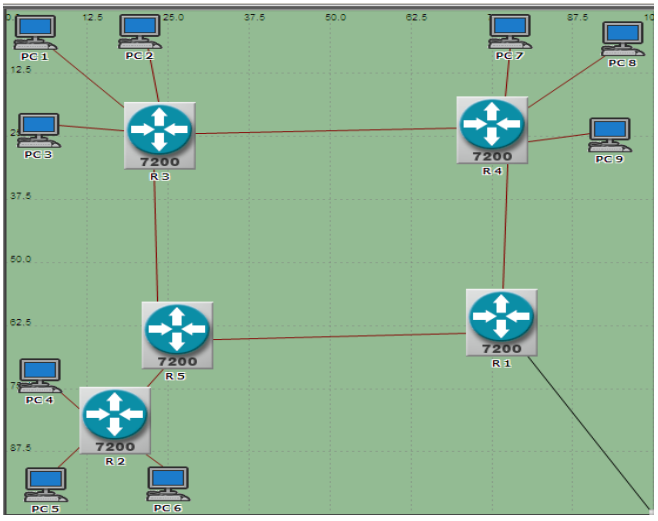

Figure 12: the subnet interior
All routers and workstations are connected together using Fast Ethernet cable (100Mbps). All 16 subnets are connected using SONET OC3 fiber cable ( $\approx 148 \mathrm{Mbps})$. In this simulation OSPF routing protocol and area division is tested by applying three applications that run simultaneously. The effect of the OSPF and the effect of dividing the network into different sub networks are simulated and analyzed. The three applications are:

- EMAIL APPLICATION

- HTTP APPLICATION

- VOICE APPLICATION

\subsection{Simulation results}

The effect of dividing areas will be analyzed for each application separately, and then a sum up will be made to give an overall result.

Figure 13 shows the overall OSPF traffic sent through the network. The figure shows three peaks. The first peak at $55 \mathrm{sec}$ shows that traffic of one area was much more than the two and four and eight areas. This traffic represents the routing traffic within individual areas

Then the second peak, it shows that the traffic of two and four and eight areas. This traffic represents the routing traffic across area gateways, and this justifies why the network which has no area configuration has no other peaks except one (Blue curve).

This is because when there are no areas configured, all the network traffic is sent only one time, while when areas are configured on the network, each area send traffic inside it (Internal LSA), then the ABR start to send summaries (External LSA) of the attached areas to the backbone. Then the ABR send OSPF traffic back representing summaries of other areas to its attached areas which is used to update the routing table within individual areas. This represents the third peak. [13]

The simulation results of running voice as one of the real time applications over OSPF based IP network to study the effect dividing the network into different OSPF area on the application quality of service. Figure 14 shows that the delay variation in case of using one area (Blue) is higher than those of using two or more areas. This is due to the reduction of IP traffic sent over the network when it is divided into sub areas.

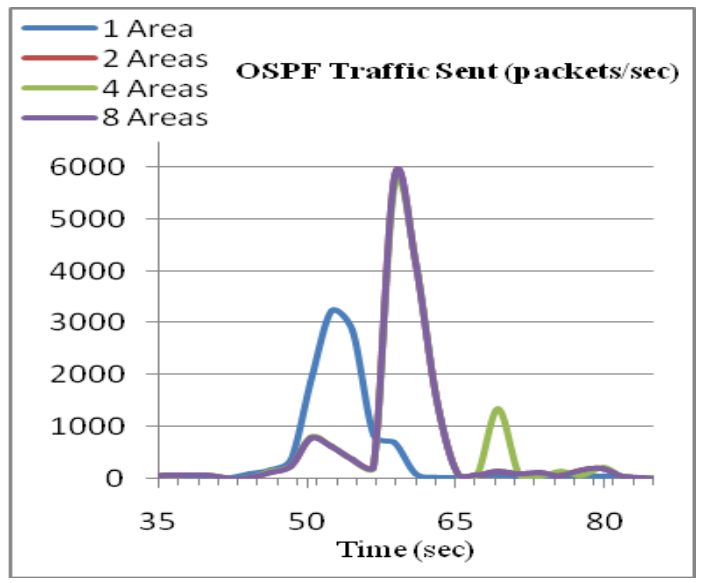

Figure 13 : OSPF traffic sent (Packets/sec) 


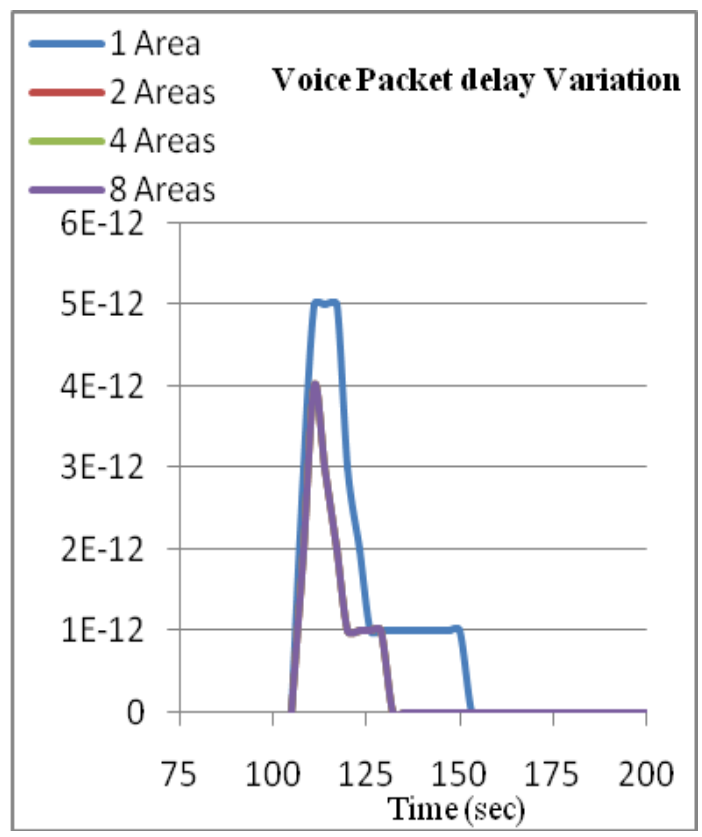

Figure 14 : Voice Packet Delay Variation

As can be seen from figure 15 and 16, by comparing the voice traffic sent and received, there is almost zero packets lost, which ensure that the quality of voice was perfect

As shown in figure 17, as the number of areas increase the amount of traffic passing through the backbone area increase, and this justifies why the convergence duration increase.

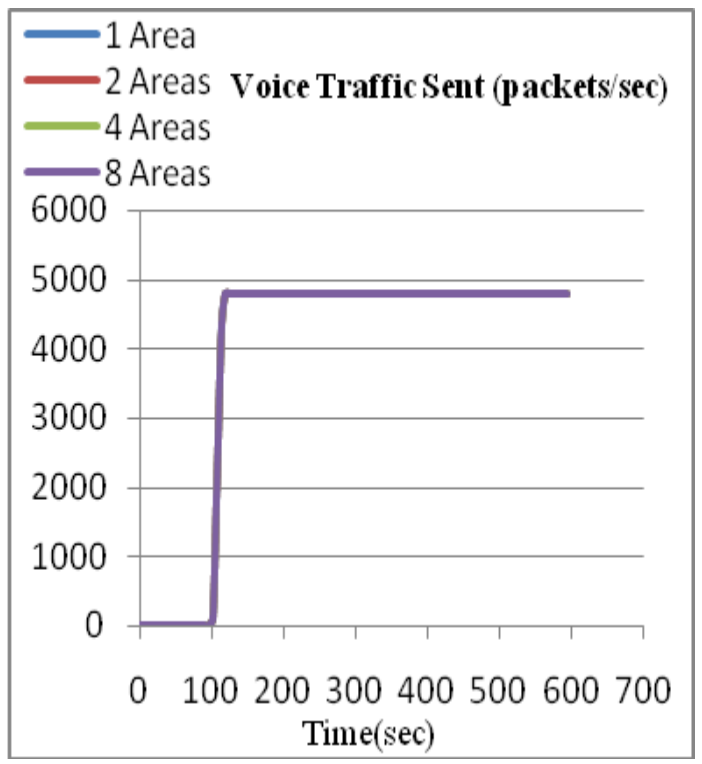

Figure 15 : Voice Traffic sent (Packets/sec)

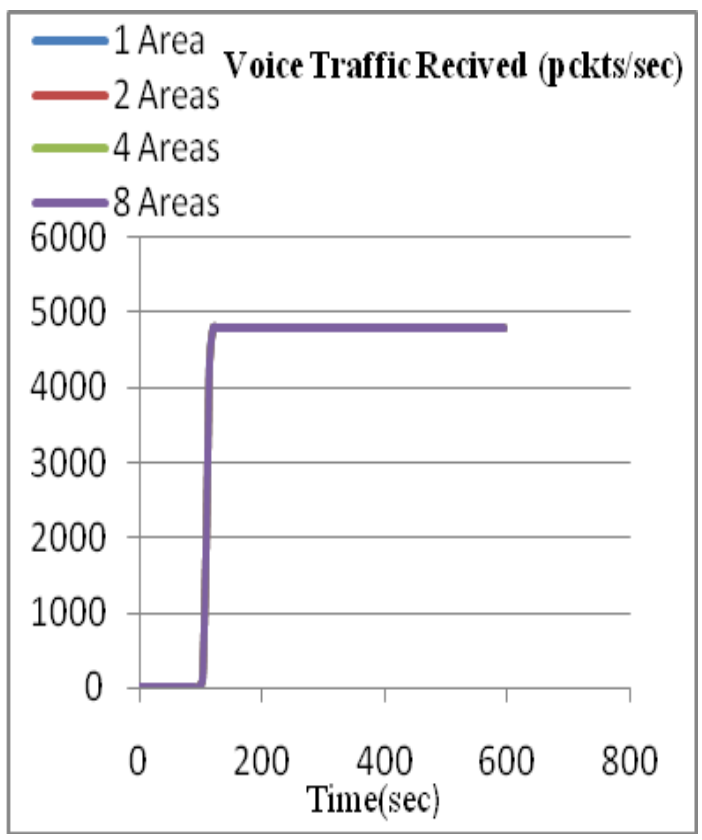

Figure 16 : Voice Traffic received (Packets/sec)

In The presence of areas, all the traffic passes through limited number of gateways which is considered bottle necks, thus the real time traffic compete with all of the other back ground traffic at these bottle necks thus the routers take more time to converge.

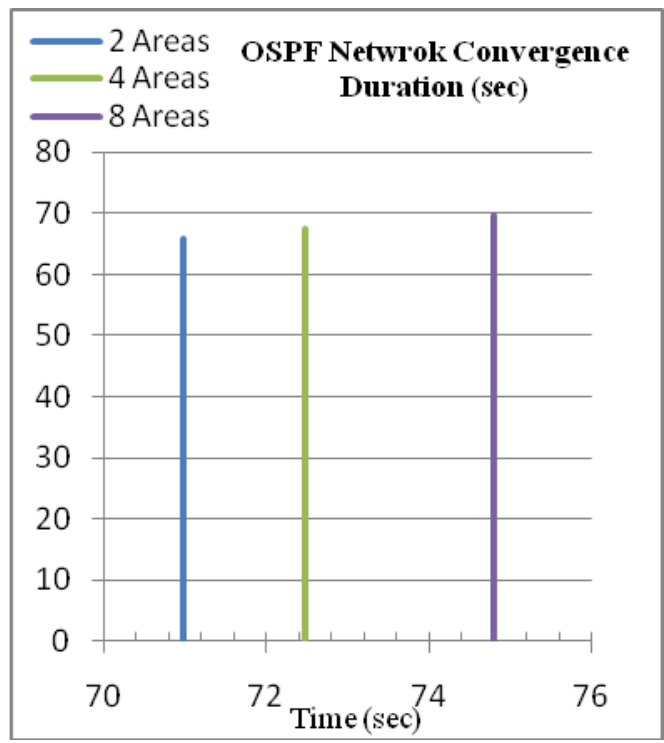

Figure 17 : Network Convergence duration (sec)

\section{CONCLUSIONS}

Interior routing protocols like EIGRP and OSPF are widely being used along with the basic protocol which is the RIP. In this paper, we have presented a comparative analysis of selected routing protocols EIGRP, OSPF and RIP. The comparative analysis has been done in the same network with different protocols for real time applications. Performance has been measured on the basis of 
some parameters that aimed to figure out the effects of routing protocols.

In network convergence parameter the EIGRP gave the best conversion duration and was the first to converge. Also it had the best reaction to node failure thus in conversion duration it is more reliable in real time applications. For the routing traffic the OSPF was the one with the most traffic sent and was the last one to send routing traffic on the other hand EIGRP was the first one to send traffic but RIP protocol had the least traffic as it sends only the number of hops. Also the EIGRP was the fastest to send routing traffic after node failure than the OSPF. For the delay variation OSPF protocol was the highest unlike the EIGRP and RIP which almost had the same values and when evaluating the end to end delay the RIP was the worst and the EIGRP was slightly better than the OSPF thus it had the least delay

Dividing an OSPF autonomous system into independent routing areas allows area topology abstraction, reducing route overhead, table size, and convergence time, while providing some isolation from bad routing data. On the contrary, areas reduce connectivity, while increasing configuration complexity, routing path length, and traffic concentration

The Interior routing protocol OSPF is widely being used in the computer networking. In this paper, we have presented a comparative analysis of different area configuration. The comparative analysis has been done on the same network with three applications. Performance has been measured on the basis of some parameters that aimed to figure out the effects of dividing the network in different areas. Network performance can be enhanced by increasing the number of areas.

Another performance metrics for real time application is packet delay variation, which measures the differences between the delays of packets. The performance of packet delay variation for 8 areas is better than 1 area. In future, a research work can be done on how to divide a main area into sub areas.

\section{REFERENCES}

[1] Tanenbaum, Andrew s. Computer Networks. s.l.: Pearson Education, 2003.

[2] Mohammad Nazrul Islam, Md. Ahsan Ullah Ashiqu, Simulation Based EIGRP over OSPF Performance Analysis, Master Thesis in Electrical Engineering Emphasis on Telecommunications Thesis no: 4983 May 14, 2010

[3] Rick Graziani and Allan Jonson, "Routing protocols and concepts: CCNA exploration companion guide" Pearson Education. London, 2008.

[4] [2] Dong (Don) Xu, OSPF, EIGRP AND RIP PEFORMANCE ANALYSIS BASED ON OPNET, ENSC835: COMMUNICATION NETWORKS, SPRING 2011

[5] Routing Overview available at: http://networking.ringofsaturn.com/IP/Routing.php

[6] Moy, John. OSPF Anatomy of an internet routing protocol. May 200.

[7] Cisco Systems, I., Cisco Networking Academy Program CCNA 1 and 2 Companion Guide Third Edition. 2003.

[8] Internetworking Technologies Handbook Fourth Edition

[9] Network Working Group at http://www.ietf.org/rfc/rfc2328.txt

[10] Berkowitz,Howard.http://www.certificationzone.com/cisco/st udyguides/component.html?module=studyguides. [Online] OSPF Part 2: Using OSPF in Hierarchical Systems.

[11] IKram Ud Din, Saeed Mahfooz and Muhammad Adnan ,Analysis of the Routing Protocols in Real Time Transmission, Global Journal of Computer Science and Technology, Vol. 10 Issue 5 Ver. 1.0 July 2010 p.18-22

[12] Mohammed A. Aabed RoutingInOPNET(30/11/2008) 